\title{
Quantitative assessment by local probe methods of the mechanical and electrical properties of inkjet-printed PEDOT:PSS thin films over Indium Tin Oxide substrates
}

\author{
E. Gutiérrez-Fernández, ${ }^{a *}$ I. A. Gabaldón-Saucedo, ${ }^{\mathrm{b}}$ M. C. García-Gutiérrez, ${ }^{\mathrm{a}}$ A. Varea, ${ }^{\mathrm{b}}$ A. \\ Nogales, ${ }^{a}$ E. Rebollar, ${ }^{c}$ A. Vilà, ${ }^{b}$ T. A. Ezquerra, ${ }^{{ }^{* * *}}$ and A. Cirera ${ }^{\mathrm{b}}$
}

anstituto de Estructura de la Materia (IEM-CSIC), Serrano 121, 28006, Madrid, Spain. ${ }^{b}$ Departament d'Enginyeria Electrònica i Biomèdica, IN2UB-Universitat de Barcelona, 08028, Barcelona, Spain.

c Instituto de Química Física Rocasolano (IQFR-CSIC), Serrano 119, 28006, Madrid, Spain. Corresponding authors: *edgar.gutierrez@iem.cfmac.csic.es; **t.ezquerra@csic.es

KEY WORDS: PEDOT:PSS, Quantitative Nanomechanical Mapping, QNM, Conducting-AFM, Inkjet printing, Thin films, ITO

\begin{abstract}
Thin films of Poly(3,4-ethylenedioxythiophene) complexed with Poly(styrenesulfonate) (PEDOT:PSS) on Indium Tin Oxide (ITO) have been prepared by inkjet printing technology. The process allows obtaining thin films over large areas. A relatively broad range of thicknesses can be obtained by the addition of subsequent polymer layers resembling an additive manufacturing process. The resulting inkjet PEDOT:PSS films are homogeneous as regard both electrical and mechanical properties. In spite of the characteristic parallel line topography of these inkjet print films, both the Quantitative Nanomechanical Mapping (QNM) and the Conductive-Atomic Force Microscope (C-AFM) characterization reveal homogeneous values throughout the polymer surface regardless of the welding zones. The results discussed in this work provide the basis for the application of inkjet printing as deposition method for electrically conducting PEDOT:PSS into a large area with mechanical stability.
\end{abstract}

\section{Introduction}

The continuous appearance of emerging technologies for the advanced processing of conducting and semiconducting polymers is facilitating the use of these materials in applications classically restricted to silicon-based devices. Among the different technologies implied in scalable additive manufacturing inkjet printing is very well-suited for processing polymer materials[1]. Inkjet printing is based on layer-by-layer deposition and arrangement of droplets of a liquid material, usually referred to as ink. By solvent 
evaporation, the solved material typically solidifies via either crystallization or vitrification subsequently to the deposition process[2]. In particular, inkjet printing electronics is envisioned as an attractive approach to produce flexible organic-based devices as an alternative to traditional rigid electronics. Inkjet printing allows the production of large, scalable films with customized shapes. This process has been applied to create a wide variety of organic electronic devices including organic solar cells [3, 4], electrochemical transistors [5-7], read-only memories [8] and sensors[9, 10] among others. Regarding conducting polymers, poly(3,4-ethylenedioxythiophene) complexed with poly(styrenesulfonate) (PEDOT:PSS) is a water-soluble material which can form conducting and transparent supported as well as free-standing thin films by using different deposition procedures including solution cast[11], spin-coating[12, 13], electro spinning[14] or inkjet[5, 15, 16] among others. For all these deposited polymer film forming techniques, the final properties may strongly depend on film uniformity $[15,17]$. In particular, the thickness uniformity of PEDOT:PSS deposited by inkjet on Indium Tin Oxide (ITO)-covered glass substrates has been shown to have a strong impact on photovoltaic performance[3, 4]. Also, surface roughness has been shown to affect significantly the electrical properties of PEDOT:PSS deposited by inkjet on glass substrates[17]. Inkjet-printed polymer layers are, in general, formed by pressure, forcing a fluid through fine nozzles forming micron size droplets. Upon reaching the substrate, the droplet forms a deposit by spreading, wetting and drying on the substrate surface[3]. Among the different substrates used for PEDOT:PSS deposition, ITO is one of the most employed materials for organic photovoltaics [18-20]. Inkjet printers eject droplets through nozzles to form lines that are typically about tens of microns[6]. After printing multiple and adjacent lines a full material film is created, and its thickness increases by the subsequent addition of layers. Similarly, as for the case of fused deposition additive manufacturing[21], the mechanical stability of the film may vary on the welding zones between adjacent lines.

In recent years, Atomic Force Microscopy (AFM) has shown to be a powerful technique[22] providing information beyond surface morphology of polymer materials but also useful for electrical[23], mechanical[24], dielectrical[25] and piezoelectrical[26, 27] properties. Soft indentation by the cantilever of an AFM is a versatile approach to study local mechanical properties of materials[28-30]. Stress-strain tests of supported PEDOT:PSS deposited by inkjet on a polyimide substrate which exhibited capabilities as strain sensor have been reported[10]. The mechanical properties of either spin-coated[31] PEDOT:PSS films supported on silicon or of self-standing PEDOT:PSS prepared by drop cast $[11,13]$ or by spin-coating[12] have also been previously discussed in the literature. Although both the morphology and the electrical properties of inkjet deposited 
PEDOT:PSS layers have been studied extensively[3, 4, 15, 17], their mechanical properties have not received much attention yet. Thus, due to the great influence of preparation variables on the features and morphology of PEDOT:PSS[3, 13], it is pertinent to attempt an evaluation of the mechanical properties of inkjet PEDOT:PSS films by local probe methods. These techniques allow us to get insights about the behaviour of the material through specific nanometric areas.

In this work, we present a study of the mechanical and electrical properties of PEDOT:PSS inkjet printed films by employing AFM local probe Quantitative Nanomechanical Mapping analysis (QNM-AFM) in combination with conducting AFM (C-AFM). Our results indicate that in spite of the line texture presented by the inkjet films the welding between lines is rather efficient providing both homogeneous Young Moduli and electrical current values.

\section{Experimental part}

\subsection{Materials and film preparation}

Indium Tin Oxide (ITO) unpatterned covered glass squares (S111 from Ossila, UK) with an area of approximately $1 \mathrm{~cm}^{2}$ were used as substrates for inkjet deposition. The substrates were sonicated in acetone for 10 minutes and then in isopropyl alcohol for other 10 minutes before using them. Subsequently, they were rinsed in deionized water and dried with nitrogen blow. A PEDOT:PSS aqueous dispersion (Heraeus Clevios ${ }^{\mathrm{TM}}$ AI 4083 from Ossila, UK, PEDOT:PSS ratio 1:6) was used to fill in a cartridge of an Inkjet printer (Dimatix 2831, Fujifilm, USA). The viscosity of the as-received PEDOT:PSS dispersion was $4.32 \mathrm{cP}$ at $10 \mathrm{rpm}$ as determined by a Cone/Plate (CPE-40) version of a Brookfield DV-I Prime Viscosimeter at room temperature. Taking into account the specifications of the printer, the viscosity value was suitable for the formation and ejection of stable droplets at room temperature. Thus, no additives were added. The droplet volume was fixed at 10 $\mathrm{pL}$. Continuous lines pattern of about $25 \mu \mathrm{m}$ width were printed forming squared layers of approximatively $1 \times 1 \mathrm{~cm}^{2}$. Samples with 1, 2, 3 and 4 layers were deposited. After inkjet printing process the samples were heat treated at $50{ }^{\circ} \mathrm{C}$ for two hours to eliminate the water solvent. The samples were stored and measured at relative humidity $(\mathrm{RH})$ and temperature values of $28 \pm 1 \%$ and $21 \pm 0.5^{\circ} \mathrm{C}$ respectively as monitored by a temperature and $\mathrm{RH}$ data logger (EL-USB-2-LCD, Lascar Electronics).

\subsection{Atomic Force Microscopy (AFM)}

AFM topography measurements were performed by a Bruker Multimode 8, equipped with a Nanoscope V controller. AFM topography images were collected in tapping mode using gold-coated silicon probes (Tap300GB-G probes by BudgetSensors). The thickness of the 
films was measured by scratching the film with a razor blade. AFM imaging of the scratched area performed perpendicularly to the scratching direction can be used to quantify the film thickness by measuring the height profile[12].

\subsection{Quantitative Nanomechanical Mapping (QNM)}

QNM measurements were performed with the same AFM equipment and using the PeakForce Quantitative Nanomechanical Mapping (PF-QNM) method. PF-QNM is a contact AFM protocol, based on the force-volume method. In these types of methods, force-distance curves are recorded by nanoindentation of the sample in a point-by-point fashion. The maximum force, referred to as peak force, is controlled at each pixel to obtain force-distance curves. In this method, the piezoscanner is oscillating at $2 \mathrm{kHz}$, while the probe remains at rest, which allows the simultaneous acquisition of force- tip-sample distances curves and topography images[24, 32, 33]. The measurements were made at ambient conditions using a standard cantilever holder. Quantitative measurements were carried out with RTESPA-300 probes from Bruker. The actual cantilever spring constant $(\mathrm{k}$, nominally $40 \mathrm{~N} / \mathrm{m})$, was measured by the thermal tune method and found to be around $15-30 \mathrm{~N} / \mathrm{m}$. Tip radius was calibrated against a polystyrene standard provided by Bruker. The measured value of the tip radius was around 6-15 nm. In every sample, images of $25 \times 25 \mu \mathrm{m}^{2}$ were acquired and then analyzed with the NanoScope Analysis 1.5 software. Young's Modulus values were obtained from the force curves (Fig. S1 from the supplementary material) according to the Derjaguin-Muller-Toporov (DMT) model[24, 34]. The DMT-Modulus was estimated for each sample as the average of the DMT-Modulus values of every pixel of a given image. The errors are calculated as their root-mean-square. The DMT-Modulus obtained in this way corresponds to the so-called reduced Young's modulus $\left(E_{r}\right)$. From the later, the Young's modulus of the sample ( $\mathrm{E}_{\text {Young }}$ ) can be estimated by using the following equation:

$$
E_{r}=\left[\frac{1-v_{s}^{2}}{E_{Y o u n g}}+\frac{1-v_{t i p}^{2}}{E_{\text {tip }}}\right]^{-1}
$$

where $E_{Y o u n g}$ and $E_{\text {tip }}$ are the Young's modulus of the sample and the tip respectively, and $v_{s}$ and $U_{\text {tip }}$ the Poisson coefficient fo sample and tip respectively[24, 29, 34]. The DMT reduced modulus can be transformed into Young's Modulus considering the Poisson coefficient[29] which for PEDOT:PPS was taken as $0.33[11,12]$. The force-distance curve analysis can be performed simultaneously with the topography, elastic modulus, adhesion force and deformation.

\subsection{Current Atomic Force Microscopy (C-AFM) mapping}

Electrical current measurements were accomplished by C-AFM by using Pt-Ir covered $\mathrm{Si}$ conductive tips with a spring constant of $\mathrm{k}=0.2 \mathrm{Nm}^{-1}$, (SCM-PIC by Bruker). A little zone of the PEDOT:PSS film was removed in order to link the ITO substrate with an iron support through conductive silver epoxy (CW2400, Chemtronics). For C-AFM measurements the equipment is used in contact mode by measuring simultaneously both topography and mapping electrical 
current through the perpendicular direction of the sample. A bias electric field is applied to the conducting substrate while the electric current is measured by a preamplifier.

\subsection{Grazing Incidence Wide Angle X-ray Scattering measurements}

Grazing Incidence Wide Angle X-ray Scattering (GIWAXS) experiments were performed at the DUBBLE beamline of the European Synchrotron Radiation Facility (ESRF, Grenoble, France) by a set-up described elsewhere[35, 36]. An X-ray wavelength of $\lambda=0.103 \mathrm{~nm}$ and a beam size of $0.6 \times 0.3 \mathrm{~mm}^{2}(\mathrm{HxV})$ was used. The GIWAXS patterns were recorded by a Frelon detector located at $121.93 \mathrm{~mm}$ from the sample position. Patterns, acquired typically with an acquisition time of $30 \mathrm{~s}$, were corrected from background scattering and analyzed by the Fit2D software[37]. The measurements were performed with grazing angles between $0.1^{\circ}$ and $0.2^{\circ}$ which are below the critical angle of ITO[38]. The scattering of the inkjet deposited polymer is very weak. Therefore the pattern of the air background, multiplied by a factor was subtracted from those of the PEDOT:PSS samples. The factor was estimated as the higher one which guarantees that no negative intensity values appear.

\section{Results and discussion}

\subsection{Inkjet deposition of PEDOT:PSS on ITO}

Fig. 1a shows the AFM tapping topography image of an area of $500 \times 500 \mathrm{~nm}^{2}$ of the one layer PEDOT:PSS inkjet sample. Roughness, calculated from Fig. 1a as the root mean square of the deviation of every point from the average height, is less than $1 \mathrm{~nm}$. The surface is quite smooth with a texture distributed in grains of 40-70 nanometers diameter. The phase image (Fig. 1b) presents a relatively high contrast among 10-50 nanometers grains and the rest of the material. Since AFM phase imaging can be sensitive to material composition, these results are compatible with a previously proposed model for spincoated PEDOT:PSS thin films[13]. According to this model, PEDOT segments arrange themselves inside entangled PSS chains forming a 'pancake' structure with the localized core of PEDOT-rich domains surrounded by a PSS shell forming grains with an average of about 30-80 nm[12, 13, 16]. Additional information about the morphology of the inkjet PEDOT:PSS thin films can be obtained by C-AFM. By this technique, electrical characterization of the PEDOT:PSS inkjet films was accomplished. Fig. 1c and 1d show contact mode topography and electrical current images respectively for a one layer film of inkjet PEDOT:PPS on ITO. The C-AFM image, Fig. 1d is consistent with this view and exhibits conducting dot-like domains of 30-80 nanometers size, randomly distributed within a less-conducting matrix. A similar morphology is observed for inkjet PEDOT:PSS films with more layers. According to Fig. 1d, the surface sample suggests a far more abundant PSS phase domain than conducting-PEDOT one dots, which is consistent with the initial PEDOT/PSS ratio of $1 / 6$. Moreover, it has been reported that during film 
deposition, PSS tends to segregate to the surface, being enhanced after exposing to a thermal annealing[39].
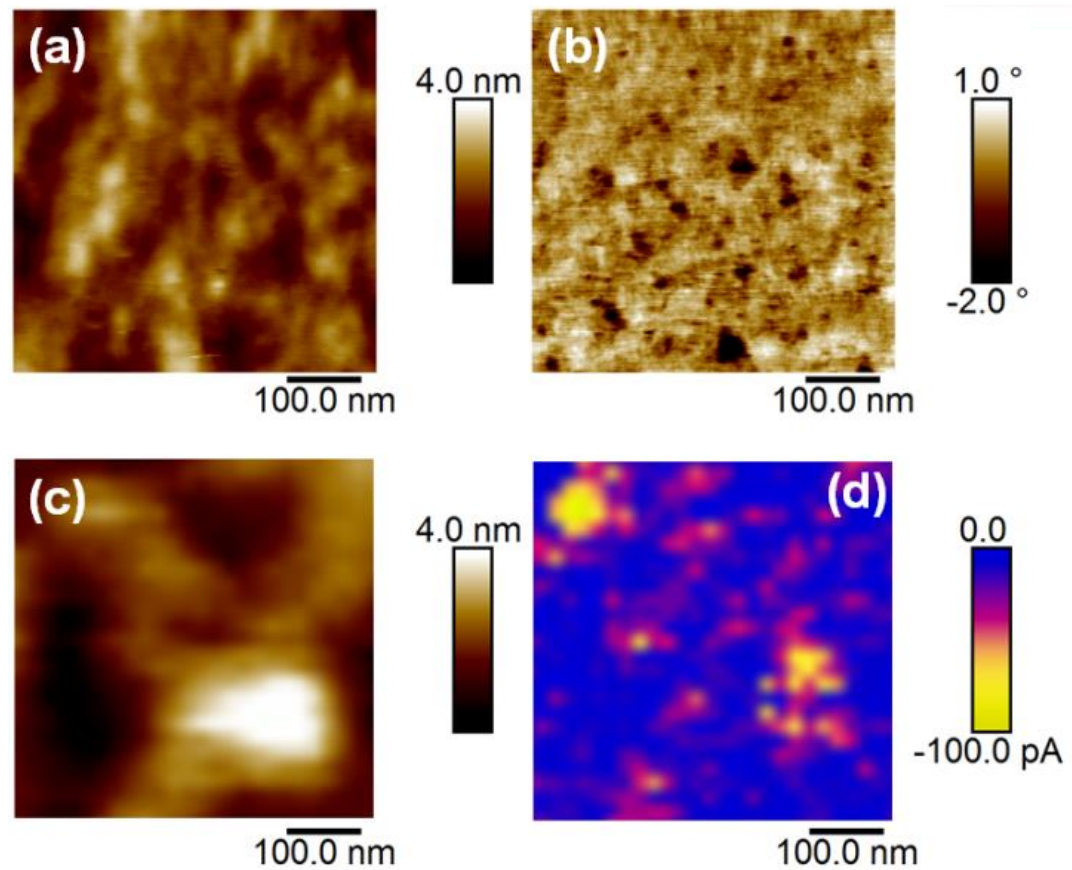

Fig. 1 (a) AFM topography image in tapping mode, (b) corresponding AFM phase image. (c) Topography contact AFM image and (d) C-AFM (bias voltage -3.0 V) for one layer PEDOT:PSS film inkjet deposited on ITO.

Fig. 2 shows, in the left column (a-d), optical microscope images of the different number of layers (from 1 to 4) of PEDOT:PSS deposited by inkjet onto ITO. A characteristic topography consisting of continuous lines is observed. The width of the lines $(\approx 25 \mu \mathrm{m})$ is closely related to the diameter of the ejected droplets. A deeper look at the higher magnification provided by the AFM is shown in the intermediate column of Fig. 2 (e-h) where AFM topographic images were acquired in a magnified area of 30x30 $\mu \mathrm{m}^{2}$. AFM reveals that the lines are rather homogenous with a roughness of about $1 \mathrm{~nm}$ estimated in an area of $1 \mathrm{x} 1 \mu \mathrm{m}^{2}$ from the AFM topography images taken at the top of one line. The height profiles are shown in the right column of Fig.2 (i-1). The thickness of the PEDOT:PSS films scales linearly with the number of inkjet deposited layers as it is shown in Fig. 3. This result emphasizes the additive character of the inkjet deposition forming PEDOT:PSS thin films. From Fig.2(i) to Fig.2(1) a curious effect in the surface of the films can be seen; the height of the printed lines seems to increase with the number of layers deposited beneath. The average distances between the maximum and the minimum point of every horizontal line of Fig.2(e-h) are represented in Fig.3. Taking into account that the quantity of material deposited in every layer step is the same, the increase of the height of these lines suggests a worst compactness between 
layers due to the appearance of interfacial capsules of air or water remaining during printing process.
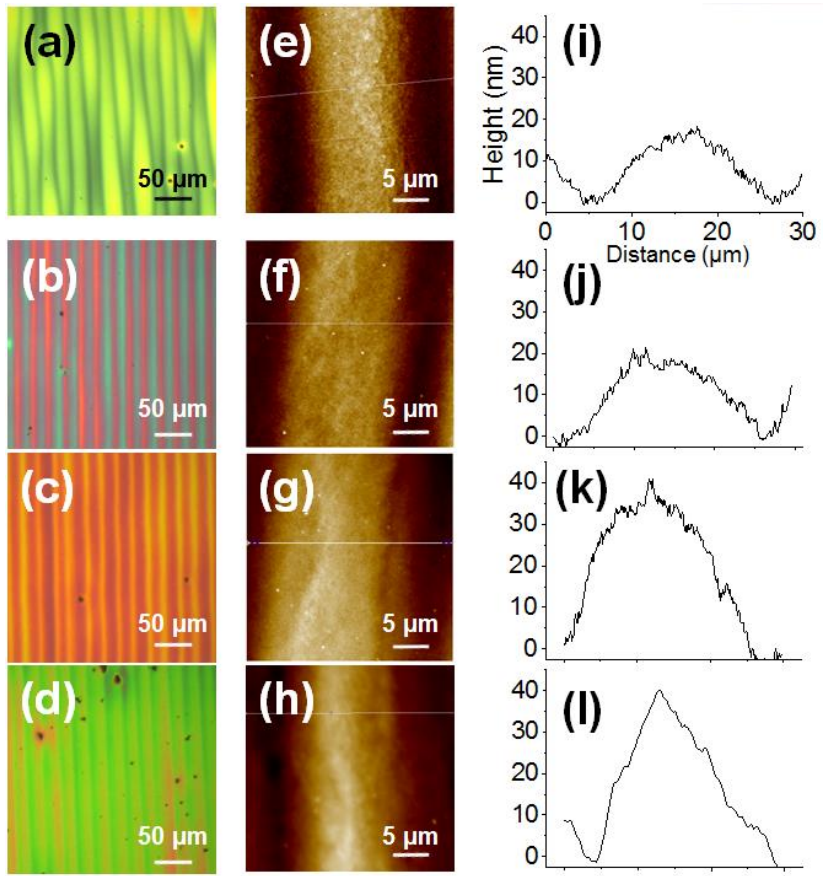

Fig. 2 (Left column) Optical microscope images for: (a) 1, (b) 2, (c) 3 and (d) 4 layers of PEDOT:PSS inkjet deposited on ITO. (Intermediate column) AFM topography images for 1 (e) to 4 (h) PEDOT:PSS layers in a magnified are $\left(30 \times 30 \mu \mathrm{m}^{2}\right)$. (Right column) AFM height profiles across the corresponding images shown in the intermediate row.

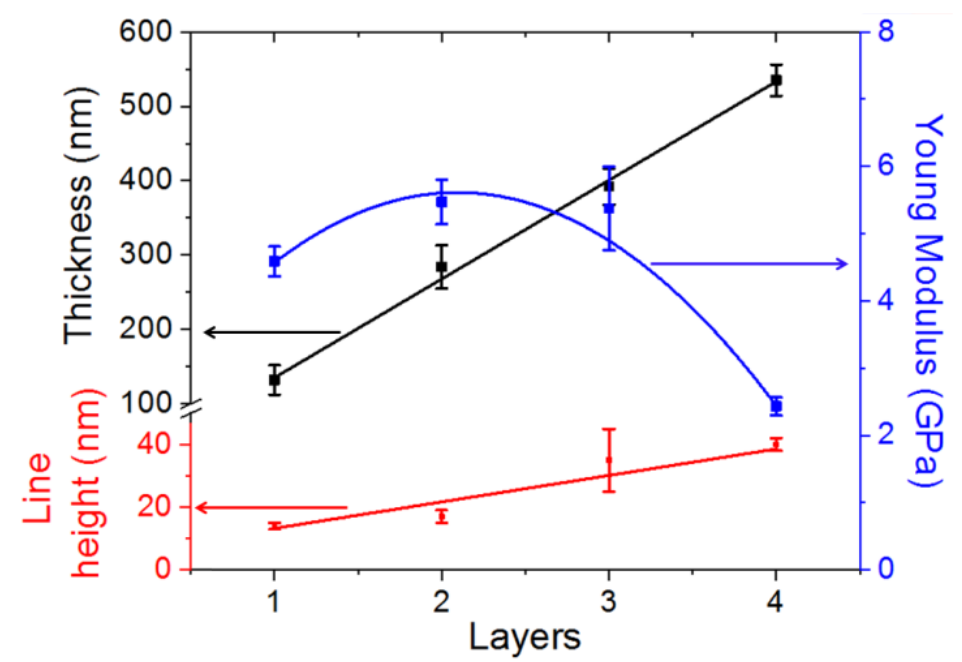

Fig. 3 (" and ', left y-axis) Thickness and height of the printed line, respectively; and (", right yaxis) Young modulus versus the number of PEDOT:PSS inkjet deposited layers over ITO. Lines and parabola for the Young Modulus data are guides for the eye. 


\subsection{Internal order of Inkjet deposited PEDOT:PSS on ITO as revealed by Grazing Incidence Wide Angle X-ray Scattering (GIWAXS)}

The inner structure of the inkjet deposited PEDOT:PSS films was examined by GIWAXS. The measurements were performed with grazing angles between $0.1^{\circ}$ and $0.2^{\circ}$ which are below the critical angle of ITO[38]. One of the issues upon investigating by GIWAXS inkjet PEDOT:PSS deposits on ITO appears from the strong diffraction features exhibited by ITO (Fig. S2b.) and the weak scattering of the inkjet samples (Fig. S2a). However at the incidence angles used for the experiments the contribution of ITO substrate to the PEDOT:PSS patterns is almost negligible (Fig. S2a). Therefore the GIWAXS patterns for the PEDOT:PSS samples were corrected for air background scattering. Fig. 4 shows background subtracted 2D GIWAXS patterns for ITO substrate and selected inkjet deposited PEDOT:PSS samples. Patterns of ITO substrate (Fig. 4a) and one layer of PEDOT:PSS (Fig. 4b) exhibit no significant scattering aside from an intense spike from the reflected beam around the beamstop which is characteristic of this type of samples [40]. When the film consists of more PEDOT:PSS layers (Fig. $4 \mathrm{c}$ and $4 \mathrm{~d}$, respectively) a scattering arc located in the meridional region starts to be detectable. GIWAXS patterns were analyzed using sector-averaged integrals to obtain intensity profiles as a function of the scattering vector q. Fig. 5 shows the plots of these intensity profiles for films with one to four PEDOT:PSS layers and for the ITO substrate.

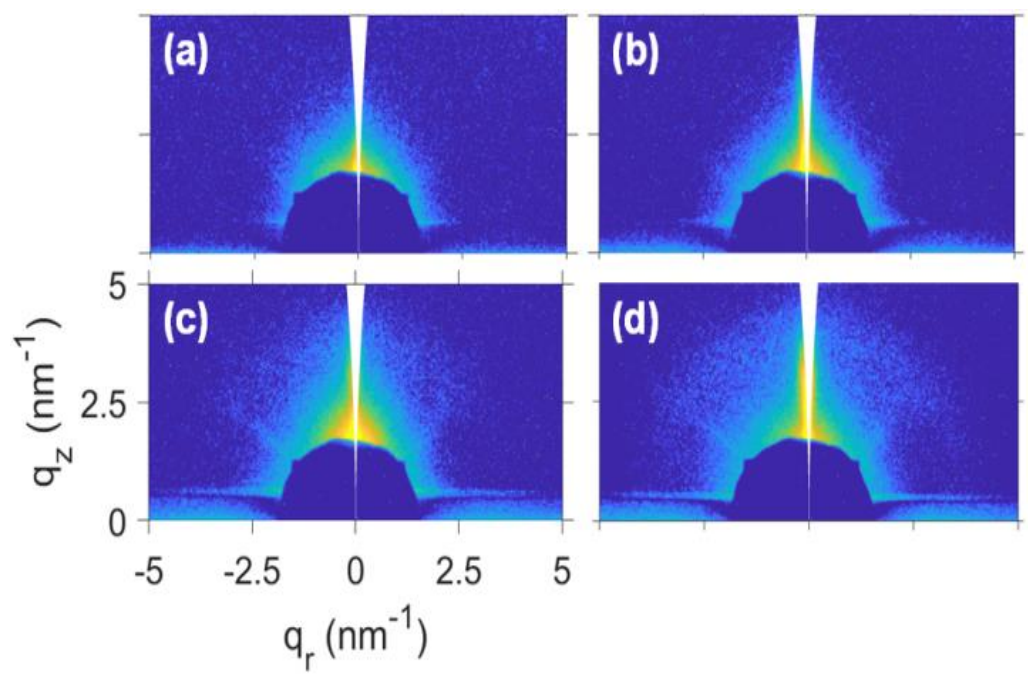

Fig. 4 2D GIWAXS logarithmic patterns taken at an incident angle of $0.2^{\circ}$ of (a) ITO-covered glass, (b) 1, (c) 2 and (d) 4 inkjet deposited layers of PEDOT:PSS. Air background has been subtracted. 


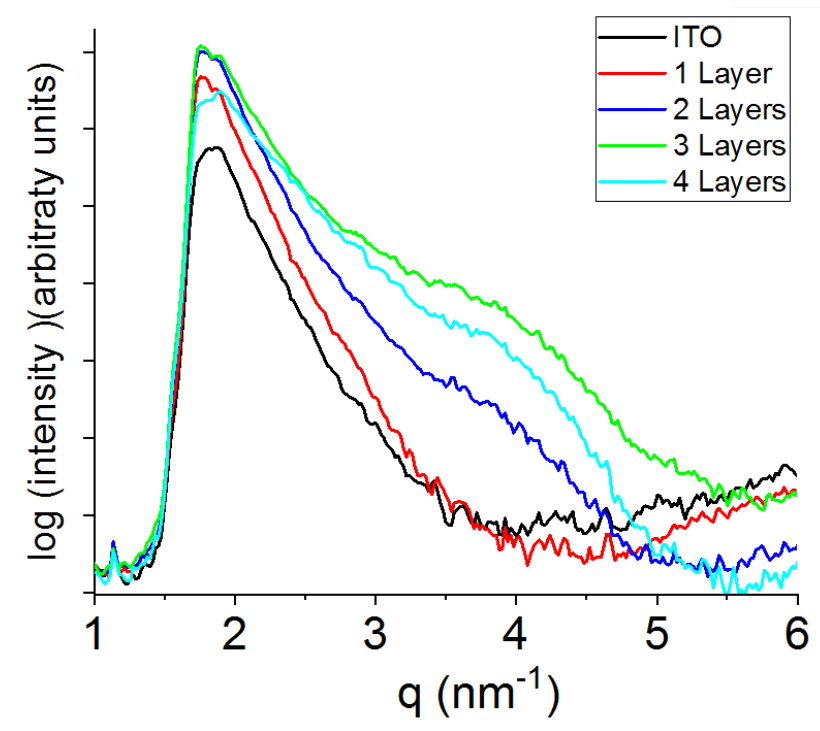

Fig. 5 Logarithm of the integrated scattered intensity as a function of the module of the scattering vector $\mathrm{q}$ for the subtracted GIWAXS patterns of ITO and 1, 2, 3 and 4 inkjet deposited layers of PEDOT:PSS on ITO.

From two to four layers the plots exhibit an excess of scattered intensity between $\mathrm{q} \approx 2.5 \mathrm{~nm}^{-1}$ and $5 \mathrm{~nm}^{-1}$ corresponding to real space distances between $\mathrm{d} \approx 2.5 \mathrm{~nm}$ and $1.3 \mathrm{~nm}$. This excess of scattering could be composed of different reflections. That corresponding to the stacking distance between lamellas of alternating PEDOT and PSS segment chains $\mathrm{d}_{(100)}$ has been reported to be $\approx$ $2.2 \mathrm{~nm}\left(\mathrm{q} \approx 2.8 \mathrm{~nm}^{-1}\right)$, coherent with the chain widths of PEDOT $(0.75 \mathrm{~nm})$ and PSS $(1.55$ $\mathrm{nm})$ [41]. In addition, reflections proposed to be associated with PSS interdigitation packing ( $\mathrm{d}=$ $\left.1.37 \mathrm{~nm}, \mathrm{q}=4.6 \mathrm{~nm}^{-1}\right)$ and with PEDOT lamellar stacking $\left(\mathrm{d}=1.4 \mathrm{~nm}, \mathrm{q}=4.5 \mathrm{~nm}^{-1}\right)$ also have been localized within this range[42]. Azimuthal integrations of the patterns between $q=3 \mathrm{~nm}^{-1}$ and $\mathrm{q}=5 \mathrm{~nm}^{-1}$ show an excess of intensity in the out-of-plane direction in comparison to that in the in-plane direction (Fig. 6). This result suggests a slightly preferential edge-on disposition of the PEDOT:PSS lamellae. 


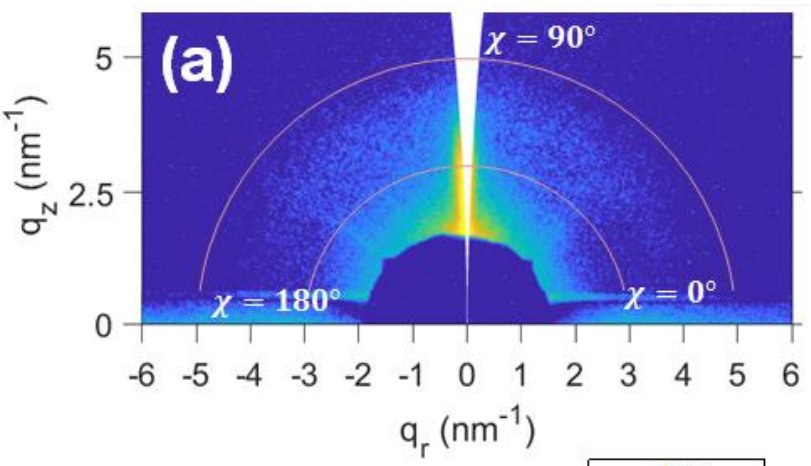

Fig. 6 (a)

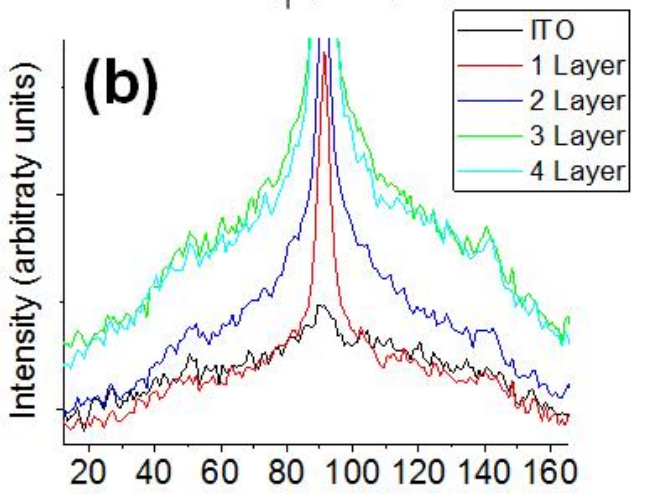

Azimuth angle $\chi\left({ }^{\circ}\right)$

GIWAXS of 4 layers of

PEDOT:PSS on ITO indicating the cake used for the azimuthal integration. (b) Azimuthal intensity integration of inkjet PEDOT:PSS samples with different layers between $q=3 \mathrm{~nm}^{-1}$ and $\mathrm{q}=5 \mathrm{~nm}^{-1}$ starting at 0 degrees in the $\mathrm{q}_{\mathrm{r}}$ positive axis, 90 degrees at the $\mathrm{q}_{\mathrm{z}}$ axis and 180 degrees at the $\mathrm{q}_{\mathrm{r}}$ negative axis.

\subsection{Mechanical properties of Inkjet deposited PEDOT:PSS on ITO}

Fig. 7a and 7b show PF-QNM images of topography and DMT modulus respectively for one PEDOT:PSS layer deposited onto ITO. The measurements were done between two consecutive inkjet printed lines including the joint region between them. It is evident that in spite of the relatively discontinuous topographical profile (Fig.7c, black data) the DMT modulus (Fig.7c blue data) remains almost in a constant value. This result indicates that the welding between two consecutive lines of inkjet deposited PEDOT:PSS is rather good providing a homogenous behaviour from the perspective of its mechanical properties. This behaviour is essentially similar for PEDOT:PSS films with more layers. 


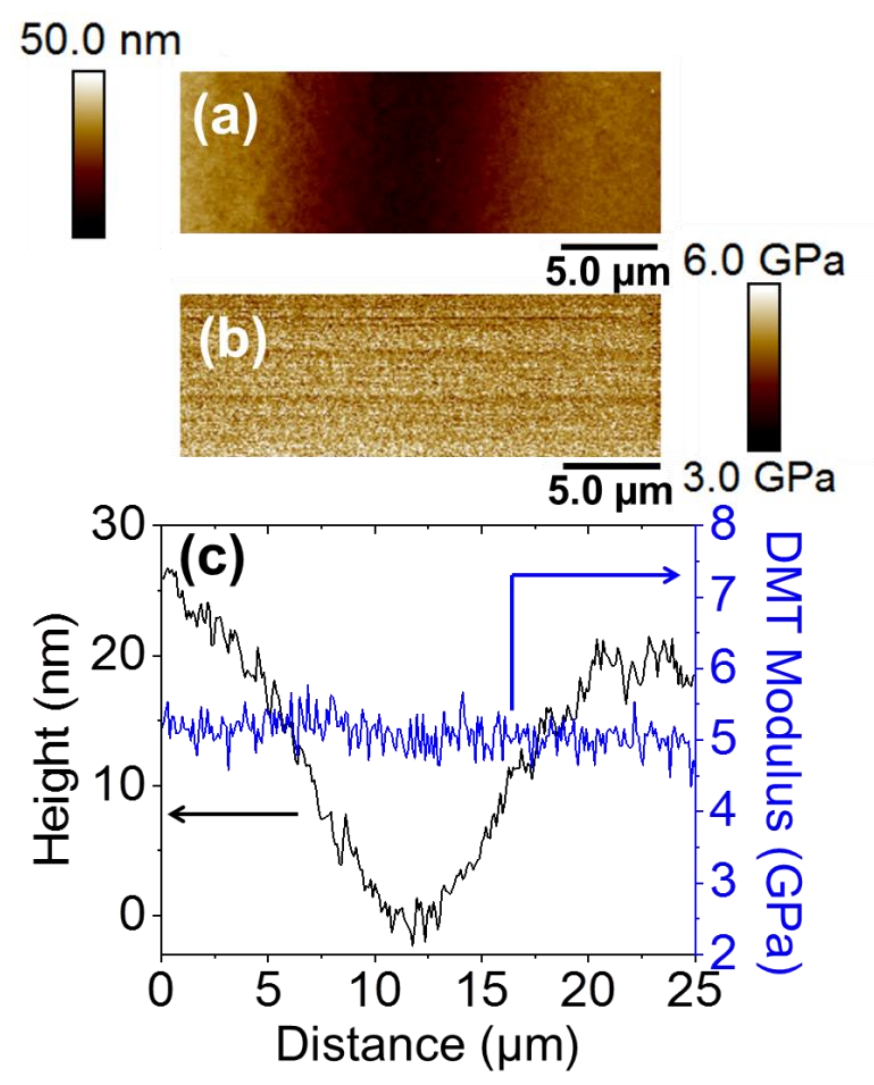

Fig. 7 (a) PF-QNM topography image and (b) DMT elastic modulus for one layer PEDOT:PSS film inkjet deposited on ITO. (c, black data) Topographical and (c, blue data) DMT Modulus profile across a direction perpendicular to the inkjet lines.

Data for Young's modulus measured for films with a different number of layers are reported in Fig. 3. The moduli values range from $4.6 \pm 0.2$ for the one layer system to $2.4 \pm 0.1$ for the four layers one reaching a maximum of $5.5 \pm 0.3$ for intermediate thick samples. These values measured for the inkjet PEDOT:PSS films compare well with the theoretical values of $3.79 \mathrm{GPa}$ $\pm 0.45 \mathrm{GPa}$ and of $4.23 \mathrm{GPa} \pm 0.50 \mathrm{GPa}$ for PEDOT and PSS respectively[43]. However, they are higher than those in the 1-3 GPa range reported for free standing thin films[11, 12, 44], microfibers of $10 \mu \mathrm{m}$ in diameter[45] and for supported films $50 \mathrm{~nm}$ thick. The overall higher values obtained here suggest that the intrinsic additive nature of the inkjet deposited films leads to a higher ionic interaction between PEDOT and PSS segments that results in a more entangled structure, with enhanced mechanical properties. This effect has been previously observed for polyelectrolyte multilayers and has been proposed to be due to the ionic bonding between the layers[46]. Due to the relatively high thickness of the films, PEDOT:PSS grains are expected to organize themselves into a pancake-like structure with improved mechanical properties[13, 47]. According to Greco et al[12], ultra-thin films with thicknesses between 30 and $80 \mathrm{~nm}$ have poorer 
mechanical properties than thicker ones because less PEDOT:PSS grains can pile up along the film thickness, yielding lower values of $\mathrm{E} \approx 1 \mathrm{GPa}$. In thicker films however, grains can distribute forming a layered 'pancake-like' structure[47], yielding improved mechanical properties and a higher Young Modulus value. Another reason to be taken into account is that in previous reports PEDOT:PSS blends with PEDOT-to-PSS ratio of 0.4-0.8 was used[12] [45] meanwhile in our case this ratio is of 0.17 . Since the theoretical value of PSS Young modulus is larger than that of PEDOT[43], it is reasonable that more PSS content in the sample renders to a higher Young Modulus average value. As the number of layers increases there is a decrease of Young Modulus (Fig. 3) that can be related with a less mechanical stability caused by a lack of compaction between layers, an effect previously hypothesized in Fig.2(i-1) and Fig.3 where an increase in the height of the printed lines suggested a lack of compactness between layers. This effect leads to a decrease of the modulus once it can counterbalance that of the ionic interaction previously described.

\subsection{Electrical properties of Inkjet deposited PEDOT:PSS on ITO}

Considering the characteristic continuous lines texture of the inkjet PEDOT:PSS films (see Fig.2) additional C-AFM measurements were carried out between two consecutive lines including the joint region between them. The results are shown in Fig. 8. Similarly as for the previously discussed case of mechanical properties, here it is also evident that in spite of the relatively discontinuous topographical profile (Fig. 8c black data) the C-AFM image and the corresponding profile (Fig. 8c blue data) renders nearly similar values. This behaviour is qualitative similar to thicker PEDOT:PSS films with more layers. This result, in conjunction with the similar one related to the mechanical properties (see Fig. 7) further support that the welding between two consecutive lines of inkjet deposited PEDOT:PSS is rather efficient and provides homogenous both mechanical and electrical properties. 


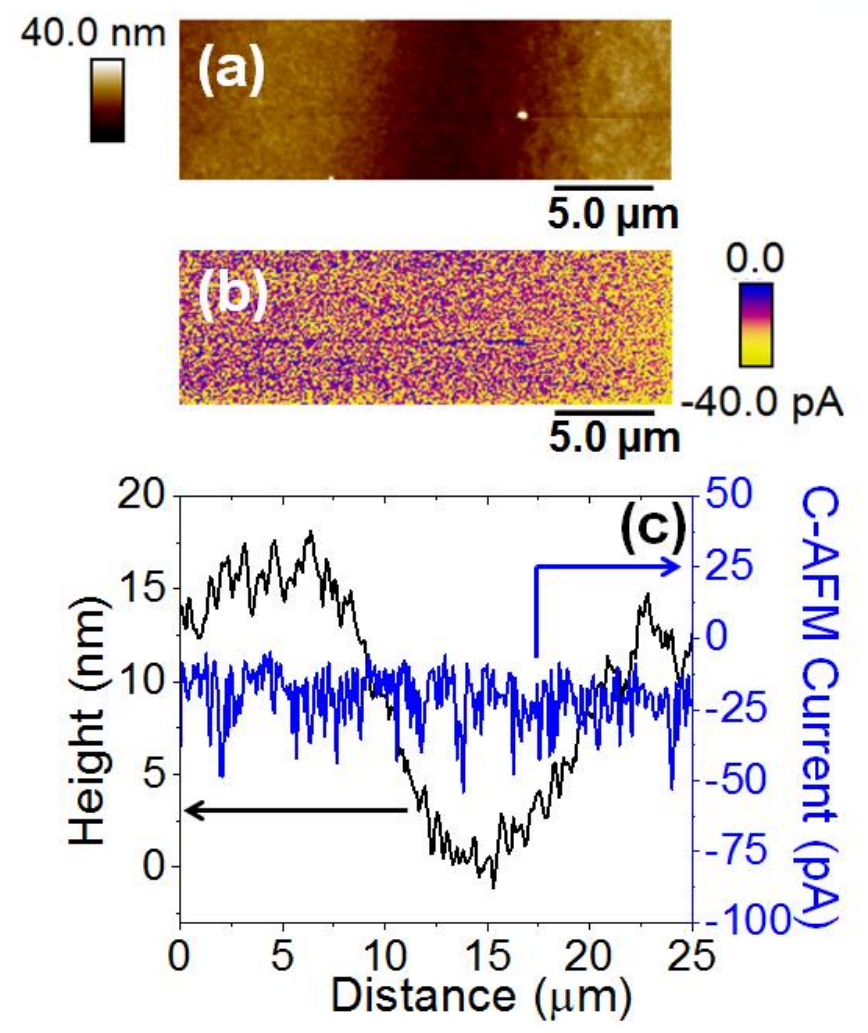

Fig. 8 (a) Contact mode AFM topography image and (b) Electrical current C-AFM image for one layer PEDOT:PSS film inkjet deposited on ITO. (c, black data) Topographical and (c, blue data) electrical current profile across a direction perpendicular to the inkjet lines.

\section{Conclusions}

The mechanical and electrical properties of inkjet deposited PEDOT:PSS on ITO substrates have been investigated by local probe methods. Large areas with a broad range of thicknesses of PEDOT:PSS on ITO can be obtained by inkjet printing with both homogeneous electrical and mechanical properties. The Young moduli of the PEDOT:PSS deposited films as revealed by AFM Quantitative Mechanical Analysis approaches the theoretical value. In spite of the line texture presented by the inkjet films the welding between lines is very efficient providing across the junction of consecutive lines both homogeneous Young Moduli and electrical current values. These results provide support for the use of inkjet methods for the extensive area processing of PEDOT:PSS in the form of thin films with adequate electrical conductivity and mechanical stability. 


\section{Conflicts of interest}

There are no conflicts to declare.

\section{Acknowledgements}

This work has been supported by Spanish MINECO under the projects MAT2014-59187R, MAT2015-66443-C02-1-R and CTQ2016-75880-P. E.R. thanks MINECO for the tenure of a Ramón y Cajal contract (No. RYC-2011-08069). We thank Daniel Hermida for valuable technical support during measurements at BM26 at ESRF (Grenoble, France).

\section{Credit Author Statement}

All authors have equally contributed to the paper.

\section{References}

[1] M. Vaezi, H. Seitz, S. Yang, A review on 3D micro-additive manufacturing technologies, International Journal of Advanced Manufacturing Technology, 67 (2013) 1721-1754.

[2] C.N. Hoth, S.A. Choulis, P. Schilinsky, C.J. Brabec, High photovoltaic performance of inkjet printed polymer: Fullerene blends, Advanced Materials, 19 (2007) 3973-+.

[3] S.H. Eom, S. Senthilarasu, P. Uthirakumar, S.C. Yoon, J. Lim, C. Lee, H.S. Lim, J. Lee, S.H. Lee, Polymer solar cells based on inkjet-printed PEDOT:PSS layer, Organic Electronics, 10 (2009) 536-542.

[4] S.H. Eom, H. Park, S.H. Mujawar, S.C. Yoon, S.-S. Kim, S.-I. Na, S.-J. Kang, D. Khim, D.Y. Kim, S.-H. Lee, High efficiency polymer solar cells via sequential inkjet-printing of PEDOT:PSS and P3HT:PCBM inks with additives, Organic Electronics, 11 (2010) 1516-1522.

[5] R. Mannerbro, M. Ranlof, N. Robinson, R. Forchheimer, Inkjet printed electrochemical organic electronics, Synthetic Metals, 158 (2008) 556-560.

[6] L. Basirico, P. Cosseddu, A. Scida, B. Fraboni, G.G. Malliaras, A. Bonfiglio, Electrical characteristics of ink-jet printed, all-polymer electrochemical transistors, Organic Electronics, 13 (2012) 244-248.

[7] M. Afonso, J. Morgado, L. Alcacer, Inkjet printed organic electrochemical transistors with highly conducting polymer electrolytes, Journal of Applied Physics, 120 (2016).

[8] S.J. Jung, A. Sou, E. Gili, H. Sirringhaus, Inkjet-printed resistors with a wide resistance range for printed read-only memory applications, Organic Electronics, 14 (2013) 699-702.

[9] Y. Seekaew, S. Lokavee, D. Phokharatkul, A. Wisitsoraat, T. Kerdcharoen, C. Wongchoosuk, Low-cost and flexible printed graphene-PEDOT:PSS gas sensor for ammonia detection, Organic Electronics, 15 (2014) 2971-2981. 
[10] M. Borghetti, M. Serpelloni, E. Sardini, S. Pandini, Mechanical behavior of strain sensors based on PEDOT:PSS and silver nanoparticles inks deposited on polymer substrate by inkjet printing, Sensors and Actuators a-Physical, 243 (2016) 71-80.

[11] U. Lang, N. Naujoks, J. Dual, Mechanical characterization of PEDOT:PSS thin films, Synthetic Metals, 159 (2009) 473-479.

[12] F. Greco, A. Zucca, S. Taccola, A. Menciassi, T. Fujie, H. Haniuda, S. Takeoka, P. Dario, V. Mattoli, Ultra-thin conductive free-standing PEDOT/PSS nanofilms, Soft Matter, 7 (2011) 10642-10650.

[13] U. Lang, E. Mueller, N. Naujoks, J. Dual, Microscopical Investigations of PEDOT:PSS Thin Films, Advanced Functional Materials, 19 (2009) 1215-1220.

[14] W. Zhao, B. Yalcin, M. Cakmak, Dynamic assembly of electrically conductive PEDOT:PSS nanofibers in electrospinning process studied by high speed video, Synthetic Metals, 203 (2015) 107-116.

[15] A.Y. Natori, C.D. Canestraro, L.S. Roman, A.M. Ceschin, Modification of the sheet resistance of ink jet printed polymer conducting films by changing the plastic substrate, Materials Science and Engineering B-Solid State Materials for Advanced Technology, 122 (2005) 231-235. [16] C.M. Palumbiny, F. Liu, T.P. Russell, A. Hexemer, C. Wang, P. Mueller-Buschbaum, The Crystallization of PEDOT:PSS Polymeric Electrodes Probed In Situ during Printing, Advanced Materials, 27 (2015) 3391-3397.

[17] P. Wilson, C. Lekakou, J.F. Watts, A comparative assessment of surface microstructure and electrical conductivity dependence on co-solvent addition in spin coated and inkjet printed poly(3,4-ethylenedioxythiophene):polystyrene sulphonate (PEDOT:PSS), Organic Electronics, 13 (2012) 409-418.

[18] R. Garcia-Valverde, J.A. Cherni, A. Urbina, Life cycle analysis of organic photovoltaic technologies, Progress in Photovoltaics, 18 (2010) 535-558.

[19] H. Zhou, L. Yang, W. You, Rational Design of High Performance Conjugated Polymers for Organic Solar Cells, Macromolecules, 45 (2012) 607-632.

[20] J. Cui, A. Rodriguez-Rodriguez, M. Hernandez, M.-C. Garcia-Gutierrez, A. Nogales, M. Castillejo, D. Mosegui Gonzalez, P. Mueller-Buschbaum, T.A. Ezquerra, E. Rebollar, LaserInduced Periodic Surface Structures on P3HT and on Its Photovoltaic Blend with PC71BM, ACS Applied Materials \& Interfaces, 8 (2016) 31894-31901.

[21] C.S. Davis, K.E. Hillgartner, S.H. Han, J.E. Seppala, Mechanical strength of welding zones produced by material extrusion additive manufacturing, Additive manufacturing, 16 (2017) 162166.

[22] G.J. Vancso, H. Schönherr, Scanning Force Microscopy of Polymers, Springer, Berlin, 2010. 
[23] A. Rodriguez-Rodriguez, E. Rebollar, M. Soccio, T.A. Ezquerra, D.R. Rueda, J. Vicente Garcia-Ramos, M. Castillejo, M.-C. Garcia-Gutierrez, Laser-Induced Periodic Surface Structures on Conjugated Polymers: Poly(3-hexylthiophene), Macromolecules, 48 (2015) 4024-4031.

[24] D.E. Martinez-Tong, A.S. Najar, M. Soccio, A. Nogales, N. Bitinis, M.A. Lopez-Manchado, T.A. Ezquerra, Quantitative mapping of mechanical properties in polylactic acid/natural rubber/organoclay bionanocomposites as revealed by nanoindentation with atomic force microscopy, Composites Science and Technology, 104 (2014) 34-39.

[25] G.A. Schwartz, C. Riedel, R. Arinero, P. Tordjeman, A. Alegria, J. Colmenero, Broadband nanodielectric spectroscopy by means of amplitude modulation electrostatic force microscopy (AM-EFM), Ultramicroscopy, 111 (2011) 1366-1369.

[26] D.E. Martinez-Tong, A. Rodriguez-Rodriguez, A. Nogales, M.-C. Garcia-Gutierrez, F. Perez-Murano, J. Llobet, T.A. Ezquerra, E. Rebollar, Laser Fabrication of Polymer Ferroelectric Nanostructures for Nonvolatile Organic Memory Devices, Acs Applied Materials \& Interfaces, 7 (2015) 19611-19618.

[27] D.E. Martinez-Tong, M. Soccio, M.C. Garcia-Gutierrez, A. Nogales, D.R. Rueda, N. Alayo, F. Perez-Murano, T.A. Ezquerra, Improving information density in ferroelectric polymer films by using nanoimprinted gratings, Applied Physics Letters, 102 (2013).

[28] H. Schönherr, G. Vancso, Scanning Force Microscopy of Polymers, Springer, Heidelberg.

[29] M.E. Dokukin, I. Sokolov, Quantitative Mapping of the Elastic Modulus of Soft Materials with HarmoniX and Peak Force QNM AFM Modes, Langmuir, 28 (2012) 16060-16071.

[30] J. Martin, M. Munoz, M. Encinar, M. Calleja, M. Martin-Gonzalez, Fabrication and Mechanical Characterization of Semi-Free-Standing (Conjugated) Polymer Thin Films, Langmuir, 30 (2014) 5217-5223.

[31] J. Liu, X. Wang, D. Li, N.E. Coates, R.A. Segalman, D.G. Cahill, Thermal Conductivity and Elastic Constants of PEDOT:PSS with High Electrical Conductivity, Macromolecules, 48 (2015) 585-591.

[32] K. Sweers, K. van der Werf, M. Bennink, V. Subramaniam, Nanomechanical properties of alpha-synuclein amyloid fibrils: a comparative study by nanoindentation, harmonic force microscopy, and Peakforce QNM, Nanoscale Research Letters, 6 (2011).

[33] B. Zhao, Y. Song, S. Wang, B. Dai, L. Zhang, Y. Dong, J. Lu, J. Hu, Mechanical mapping of nanobubbles by PeakForce atomic force microscopy, Soft Matter, 9 (2013) 8837-8843.

[34] B.V. Derjaguin, V.M. Muller, Y.P. Toporov, EFFECT OF CONTACT DEFORMATIONS ON ADHESION OF PARTICLES, Journal of Colloid and Interface Science, 53 (1975) 314-326. [35] W. Bras, I.P. Dolbnya, D. Detollenaere, R. van Tol, M. Malfois, G.N. Greaves, A.J. Ryan, E. Heeley, Recent experiments on a combined small-angle/wide-angle X-ray scattering beam line at the ESRF, Journal of Applied Crystallography, 36 (2003) 791-794. 
[36] E. Rebollar, D.R. Rueda, I. Martin-Fabiani, A. Rodriguez-Rodriguez, M.-C. GarciaGutierrez, G. Portale, M. Castillejo, T.A. Ezquerra, In Situ Monitoring of Laser-Induced Periodic Surface Structures Formation on Polymer Films by Grazing Incidence Small-Angle X-ray Scattering, Langmuir, 31 (2015) 3973-3981.

[37] A. Hammersley, http://www.esrf.eu/computing/scientific/FIT2D/, 2004.

[38] L.L. Yang, D.T. Ge, X.D. He, F. He, Y.B. Li, S. Zhang, Grazing incidence X-ray scattering study of sol-gel derived indium tin oxide thin films, Thin Solid Films, 517 (2009) 5151-5156.

[39] P.C. Jukes, S.J. Martin, A.M. Higgins, M. Geoghegan, R.A.L. Jones, S. Langridge, A. Wehrum, S. Kirchmeyer, Controlling the surface composition of poly(3,4-ethylene dioxythiophene)poly(styrene sulfonate) blends by heat treatment, Advanced Materials, 16 (2004) 807-+.

[40] G. Wang, W. Huang, N.D. Eastham, S. Fabiano, E.F. Manley, L. Zeng, B.H. Wang, X.A. Zhang, Z.H. Chen, R. Li, R.P.H. Chang, L.X. Chen, M.J. Bedzyk, F.S. Melkonyan, A. Facchetti, T.J. Marks, Aggregation control in natural brush-printed conjugated polymer films and implications for enhancing charge transport, Proc. Natl. Acad. Sci. U. S. A., 114 (2017) E10066E10073.

[41] N. Kim, B.H. Lee, D. Choi, G. Kim, H. Kim, J.R. Kim, J. Lee, Y.H. Kahng, K. Lee, Role of Interchain Coupling in the Metallic State of Conducting Polymers, Physical Review Letters, 109 (2012).

[42] N. Massonnet, A. Carella, A. de Geyer, J. Faure-Vincent, J.P. Simonato, Metallic behaviour of acid doped highly conductive polymers, Chemical Science, 6 (2015) 412-417.

[43] D. Tahk, H.H. Lee, D.Y. Khang, Elastic Moduli of Organic Electronic Materials by the Buckling Method, Macromolecules, 42 (2009) 7079-7083.

[44] U. Lang, J. Dual, Mechanical properties of the intrinsically conductive polymer poly(3,4ethylenedioxythiophene) poly(styrenesulfonate) (PEDOT/PSS), in: S.W. Nam, Y.W. Chang, S.B. Lee, N.J. Kim (Eds.) Mechanical Behavior of Materials X, Pts 1and 2, Trans Tech Publications Ltd, Durnten-Zurich, 2007, pp. 1189-+.

[45] H. Okuzaki, M. Ishihara, Spinning and characterization of conducting microfibers, Macromolecular Rapid Communications, 24 (2003) 261-264.

[46] P.V. Pavoor, A. Bellare, A. Strom, D.H. Yang, R.E. Cohen, Mechanical characterization of polyelectrolyte multilayers using quasi-static nanoindentation, Macromolecules, 37 (2004) 48654871.

[47] A.M. Nardes, M. Kemerink, R.A.J. Janssen, J.A.M. Bastiaansen, N.M.M. Kiggen, B.M.W. Langeveld, A.J.J.M. van Breemen, M.M. de Kok, Microscopic understanding of the anisotropic conductivity of PEDOT : PSS thin films, Advanced Materials, 19 (2007) 1196-+. 


\section{Supplementary information}

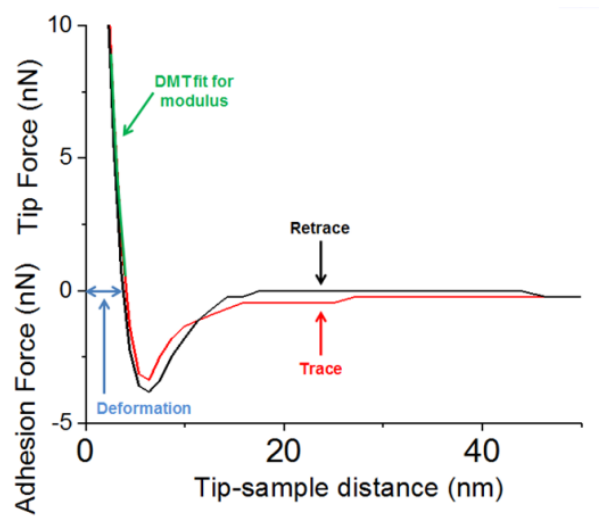

Fig. S1 Force-distance curve measured in a point of the sample with one layer of PEDOT:PSS. The red curve corresponds to the trace and the black curve to the retrace.

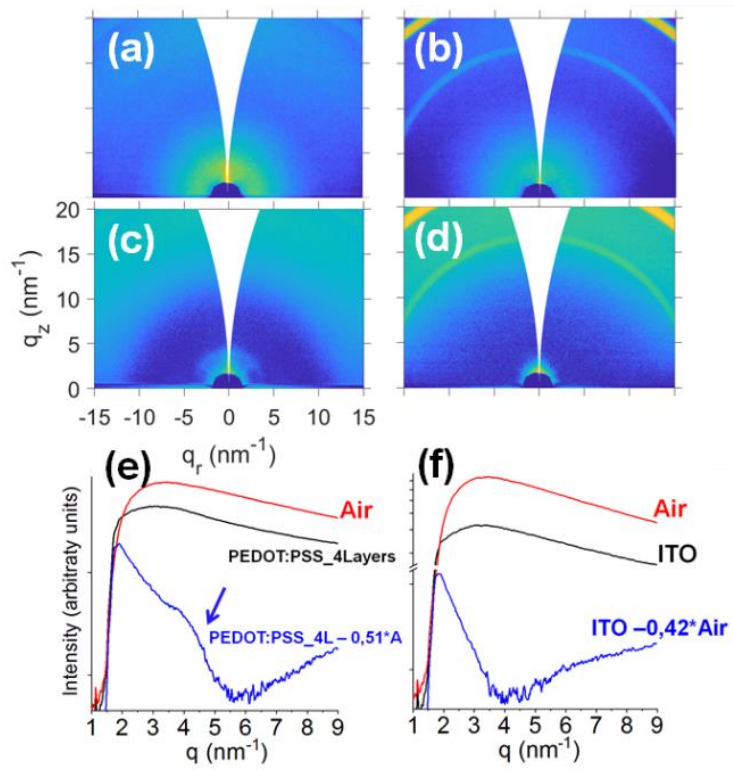

Fig. S2 GIWAXS patterns of (a) 4 layers of inkjet deposited PEDOT:PSS, (b) ITO substrate, (c) and (d) 4 layers of inkjet deposited PEDOT:PSS and ITO substrate with air background scattering subtracted. All patterns were taken at an incident angle of $0.2^{\circ}$. (e) Logarithm of the Integrated scattered intensity at low q-values for the air background, the 4 layers sample and its airsubtraction (PEDOT:PSS_4Layers - A*air). The coefficient A indicates the number which multiplies the air scattering pattern for the subtraction. The arrow on (e) indicates the excess of scattering observed for the PEDOT:PSS sample. (f) Integrated scattered intensity at low q-values for the air background, the ITO substrate and its air-subtraction (ITO - B*air). The coefficient B 
indicates the number which multiplies the air scattering pattern for the subtraction. Note that the excess of scattering present in the PEDOT:PSS samples is absent for the ITO. 